\title{
Analytical Evaluation of Various Frequency Reuse Schemes in Cellular OFDMA Networks
}

\author{
Philippe Godlewski \\ TELECOM ParisTech \& CNRS \\ LTCl \\ 46, rue Barrault \\ 75013 Paris, France \\ godlewski@enst.fr
}

\author{
Masood Maqbool \\ TELECOM ParisTech \& CNRS \\ LTCl \\ 46, rue Barrault \\ 75013 Paris, France \\ maqbool@enst.fr \\ Jean-Marc Kélif \\ France Telecom R\&D \\ Issy-les-Moulineaux, France \\ jeanmarc.kelif@orange- \\ ftgroup.fr
}

\author{
Marceau Coupechoux \\ TELECOM ParisTech \& CNRS \\ LTCl \\ 46, rue Barrault \\ 75013 Paris, France \\ coupechoux@enst.fr
}

\begin{abstract}
In this paper, we present an analytical solution to carry out performance analysis of various frequency reuse schemes in an OFDMA based cellular network. We study the performance in downlink in terms of signal to interference (SIR) ratio and cellular capacity. Analytical models are proposed for integer frequency reuse (IFR), fractional frequency reuse (FFR) and two level power control (TLPC) schemes. These models are based on a fluid model originally proposed for CDMA networks. The modeling key of this approach is to consider the discrete base stations entities as a continuum. To validate our approach, Monte Carlo simulations are carried out. Results of validation study show that results obtained through our analytical method are in conformity with those obtained through simulations. However, compared to time consuming simulations, our model is very time efficient. We also present a comparison between above three frequency reuse scheme.
\end{abstract}

\section{Keywords}

OFDMA, Fluid Model, SINR, IFR, FFR

\section{INTRODUCTION}

Orthogonal frequency division multiple access (OFDMA) is a promising multiple access technique being proposed for next generation mobile networks. The underlying technology for OFDMA based systems is orthogonal frequency division multiplexing (OFDM). With OFDM, available spectrum is split into a number of parallel orthogonal narrowband subcarriers. These subcarriers can be independently assigned to different users in a cell. Resources of an OFDMA

Permission to make digital or hard copies of all or part of this work for personal or classroom use is granted without fee provided that copies are not made or distributed for profit or commercial advantage and that copies bear this notice and the full citation on the first page. To copy otherwise, to republish, to post on servers or to redistribute to lists, requires prior specific permission and/or a fee.

ValueTools 2008 October 21 - 23, 2008, Athens, GREECE.

Copyright (C) 2008 ICST ISBN\# 978-963-9799-31-8. system occupy place both in time (OFDM symbols) and frequency (subcarriers) domains thus introducing both the time and frequency multiple access [7].

Co-channel interference (CCI) limits the capacity of an integer frequency reuse (IFR) reuse 1 cellular network (cf. section 3 for an introduction on IFR). The CCI becomes more critical for the users present in the border area of a cell. To combat this problem in an OFDMA based system, fractional frequency reuse (FFR) has been proposed in [1]. In FFR, cell is divided into inner (close to base station) and outer (border area) regions. Available bandwidth is divided among inner and outer regions in a way that former employs reuse 1 while the latter applies frequency reuse 3 . Hence, users located in cell border mitigate CCI because of frequency reuse 3. By properly adjusting the sizes of inner and outer regions, both capacity and signal to interference (SIR) can be improved.

Authors of [8] studied the performance of FFR for 3GPP/ 3GPP2 OFDMA systems and call it soft frequency reuse. Authors have used system level simulation (SLS) in their analysis. In [10] and [9], author has studied the FFR in a IEEE 802.16 based system. Author has proposed an interference coordination system, which focuses on the scheduling of users. Proposed algorithm is used in SLS to present results. Two new algorithms, fractional time reuse (FTR) and fractional time and frequency reuse (FTFR), are proposed in [2] to cater for reduced capacity in the border area of cell because of FFR. In [13], authors have studied the capacity of a WiMAX system in presence of FFR. In [3] also, performance of a FFR system is analyzed through simulations.

In contrast to existing work, in this paper we present approximate analytical models for IFR and FFR schemes of an OFDMA based cellular network. We derive expressions to calculate SIR at a given distance from base station (BS) and compute capacity using Shannon's classical formula. An expression for total data rate in the cell is also determined, when scheduling is assumed to be fair in throughput.

This paper extends the framework proposed in [4] and [5] that provides a simple closed-form formula for the other-cell interference factor $f$ on the downlink of CDMA networks as a function of the distance to the BS, the path-loss exponent, 
the distance between two BS, and the network size. The modeling key of this approach is to consider the discrete BS entities of a cellular network as a continuum.

Rest of the paper is organized as follows: section 2 introduces notations used throughout the paper and recalls the main result of the fluid model. Section 3 focuses on IFR and derives SIR and capacity expressions for both reuse 1 and reuse $K$. The case of FFR is studied in section 4. A two level power control scheme used in conjunction with FFR is considered in section 5 . In section 6 , three frequency reuse schemes (IFR, FFR and TLPC) are compared in terms of SINR, capacity and total cell data rate. Finally, section 7 discusses the conclusion of this analysis.

\section{FLUID MODEL AND NOTATIONS}

In this section, we explain the application of fluid model to an OFDMA system. We focus on the downlink and consider a single sub-carrier. BS have omni-directional antennas, such that one BS covers a single cell. If a user $u$ is attached to a station $b$ (or serving BS), we write $b=\psi(u)$.

The propagation path gain $g_{b, u}$ designates the inverse of the pathloss $p l$ between station $b$ and user $u, g_{b, u}=1 / p l_{b, u}$. In the rest of this paper, we assume that $g_{b, u}=A r_{b, u}^{-\eta}$, where $A$ is a constant, $r_{b, u}$ is the distance between BS $b$ and user $u$ and $\eta(>2)$ is the path-loss exponent.

Before presenting the expression of fluid model, we establish the following terms:

- $P_{T x}$ is the transmitted power per sub-carrier. We assume that the output power per sub-carrier is constant. Only in section 5 , we consider two possible values of output power per sub-carrier: $P_{i}$ for the inner region of the cell and $P_{o}$ for the outer region;

- $S_{b, u}=P_{T x} g_{b, u}$ is the useful power received at user $u$ from station $b$ (for traffic data);

- $W$ is the total system bandwidth and $W_{u}$ is the bandwidth dedicated to user $u$;

- $R, R_{c}$ and $R_{n w}$ are respectively the cell radius, half distance between base stations and network range (see Fig.1);

- $\rho_{u}, \rho_{B S}$ and $N_{u}$ are respectively the user density, BS density and number of users per cell.

- $D_{u}$ is the data rate allocated to a user and $D_{T}$ is the total cell data rate, such that $D_{T}=D_{u} N_{u}$

- $N_{B S}$ represents the total number of base stations in the network.

- Shannon capacity in bps/Hz is a measure of spectral efficiency.

The total amount of power experienced by a user $u$ in a cellular system can always be split up into three parts: useful signal $\left(S_{b, u}\right)$, interference and noise (Noise). It is common to split the system power into two terms: $I_{u}=I_{i n t, u}+I_{e x t, u}$, where $I_{i n t, u}$ is the internal (or own-cell) received power and $I_{e x t, u}$ is the external (or other-cell) interference. We consider that useful signal $S_{b, u}$ is included in the $I_{i n t, u}$. It should be noted that this useful signal power has to be distinguished from the commonly considered own-cell interference. In a

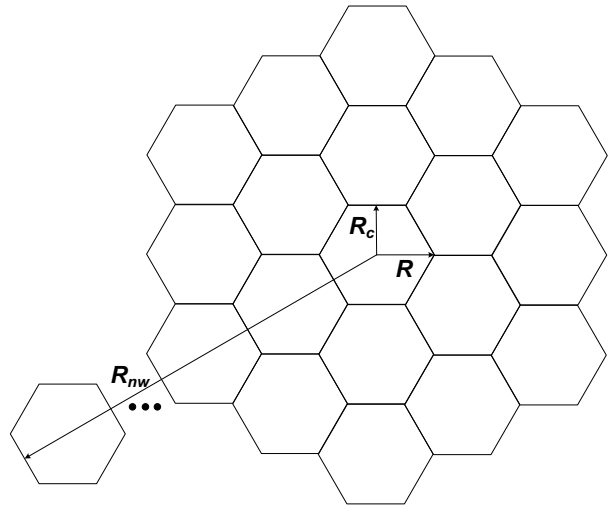

Figure 1: Hexagonal network and main parameters of the study.

CDMA network, the lack of orthogonality induces own-cell interference. In a OFDMA network, there is a perfect orthogonality between users and thus $I_{i n t, u}=S_{b, u}$.

With the above notations, reference [4] has defined the interference factor in $u$, as the ratio of total power received from other BS to the total power received from the serving BS $\psi(u): f_{u}=I_{e x t, u} / I_{i n t, u}$. The quantities $f_{u}, I_{e x t, u}$, and $I_{i n t, u}$ are location dependent and can thus be defined at any location $x$ as long as the serving BS is known. In an OFMDA network, $I_{e x t, u}$ is the total interference, and thus $f_{u}$ is the inverse of the signal to interference ratio (SIR) per subcarrier. Throughout this paper, we shall neglect noise in our analytical calculations. This is a common assumption for macro-cells in dense urban areas. In this case, the signal to interference plus noise ratio (SINR), $\gamma_{u}$ can be approximated by the SIR:

$$
\begin{aligned}
\gamma_{u} & \approx \frac{S_{b, u}}{I_{e x t, u}} \\
& =1 / f_{u} .
\end{aligned}
$$

As a consequence, it is clear that the approach developed in [4] can be adapted to OFDMA networks, as soon as the orthogonality factor $\alpha$ considered in CDMA networks is zero (details on fluid model are given in appendix). In this case, SIR per sub-carrier is simply the inverse of the interference factor considered in [4].

$$
\gamma_{u}=\frac{r_{u}^{-\eta}(\eta-2)}{2 \pi \rho_{B S}\left(2 R_{c}-r_{u}\right)^{2-\eta}} .
$$

Note that the shadowing effect is neglected in this paper. An extension of the fluid model has been proposed in [6] to take into account shadowing. The results presented in this paper can thus be extended accordingly.

We now compare the results obtained with Eq.1 with those obtained numerically by Monte Carlo simulations. The simulator assumes an homogeneous hexagonal network made of several rings around a central cell. Fig.1 shows an example of such a network with the main parameters involved in the study.

Fig.2 shows the simulated SINR (using Monte Carlo simulations) as a function of the distance from the base station. Following are the simulation parameters: $R=1 \mathrm{Km}, \eta$ between 2.7 and $4, \rho_{B S}=\left(3 \sqrt{3} R^{2} / 2\right)^{-1}$, the number of rings 


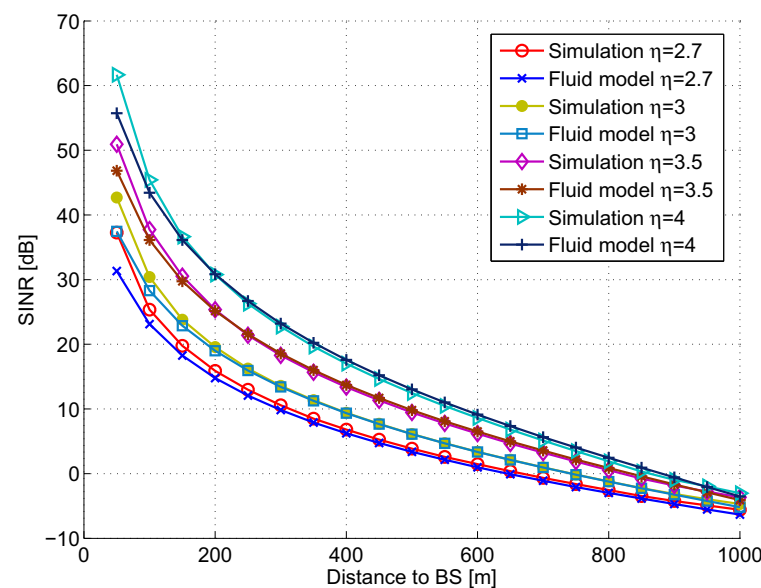

Figure 2: SINR versus distance to the BS; comparison of the fluid model with simulations on an hexagonal network with $\eta=2.7,3,3.5$, and 4 (reuse 1).

is 15 , and the number of snapshots is 3000 . To include the effect of pathloss, Erceg model [14] is used. Thermal noise density has been taken as $-174 \mathrm{dBm} / \mathrm{Hz}$ and a subcarrier spacing of $10.9 \mathrm{KHz}$ is considered [12]. Eq.1 is also plotted for comparison.

In all cases, the fluid model matches very well the simulations in an hexagonal network for various values of path-loss exponent. Only in a short area around the BS, the fluid model is a little bit pessimistic, but this is not for operator a region of great interest. It is to be noted that thermal noise was not considered in the fluid model while simulator does include its effect. However, the results of the two (fluid model and simulator) still match. It indicates that value of interference is much more pronounced as compared to that of thermal noise. Hence, neglecting thermal noise in the fluid model is a reasonable assumption.

For the rest of the paper, all above parameters are used for simulations. However, instead of different values of $\eta$, a fixed value of three is used in rest of the simulations except mentioned otherwise. The closed-form formula 1 will allow us to quickly compute performance parameters of an OFDMA network and in particular compare different reuse pattern schemes.

\section{INTEGER FREQUENCY REUSE (IFR)}

In this section, we consider the application of the fluid model to IFR environments. In integer frequency reuse, all subcarriers allocated to a cell can be used anywhere in the cell without any specification of user's location. However, reutilization of subcarriers in network cells may be one or greater. An example of IFR with frequency reuse 1 is shown in Fig.3, where $W$ represents the available network bandwidth. For frequency reuse 1, cell bandwidth equals network bandwidth.

Two cases, frequency reuse 1 and $\mathrm{K}$, have been considered. We first derive SINR and Shannon capacity expressions as functions of the distance from the BS using the fluid model. We then assume that the scheduling guarantees throughput fairness among users and we derive the total cell data rate

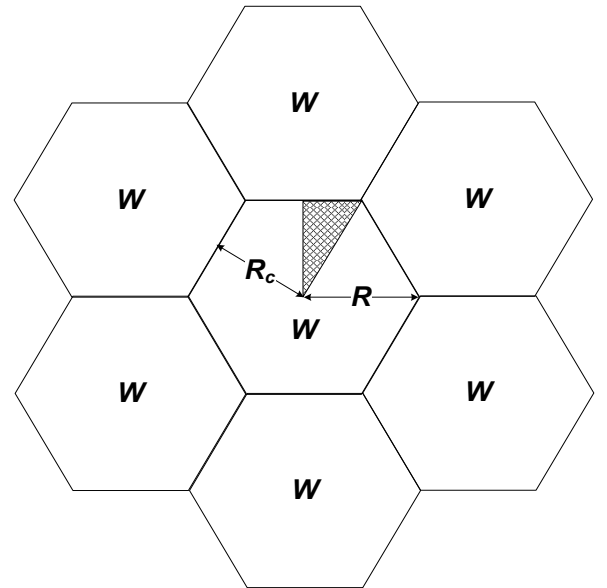

Figure 3: Integer Frequency Reuse (IFR) case (reuse 1). Shaded triangular region shows the basic integration area.

under this assumption. Results of analytical expressions are also compared to results of Monte Carlo simulations.

\subsection{IFR with Reuse 1}

Consider Eq.1 that gives expression for SINR of a subcarrier for a user at distance $r_{u}$. Since SINR, capacity and $r$ (distance from BS) are always specific to a user, in rest of the paper, subscript $u$ is omitted for these three parameters. With $\rho_{B S}=1 /\left(2 \sqrt{3} R_{c}^{2}\right)$ and introducing the normalized distance $x$ such that $x=r / R_{c}$, the expression for SINR can be rewritten as:

$$
\gamma_{I F R 1}(x)=\frac{\sqrt{3}}{\pi}(\eta-2)(2-x)^{-2}(2 / x-1)^{\eta} .
$$

By using Shannon's capacity formula, capacity (in bps/Hz) as a function of variable $x$ is given as:

$$
C_{I F R 1}(x)=\log _{2}\left[1+\gamma_{I F R 1}(x)\right],
$$

where $\gamma_{I F R 1}(x)$ is furnished by Eq.2.

We now assume that a scheduler guarantees throughput fairness among users of a cell. Users are assigned the bandwidth in a way that resultant data rate for every user, $D_{u}$, is the same. Higher the distance of a user from the BS, lower is the available capacity and thus, higher is the bandwidth (or number of sub-carriers) allocated to it. As SINR and capacity depend on $r$, let $W_{u}(r)$ be the bandwidth allocated by the scheduler to a user at distance $r$ from the BS.

User data rate, $D_{u}$, can now be written for any $r$ :

$$
D_{u}=W_{u}(r) C(r),
$$

under the constraint that total cell bandwidth $W$ cannot be exceeded, i.e.:

$$
W=\iint_{\text {cell area }} W_{u}(r) \rho_{u} d s .
$$

Integration is done over the shaded triangular region in Fig.3 and multiplied by twelve to obtain the result over the entire hexagon cell. Total bandwidth used in a cell is thus given 


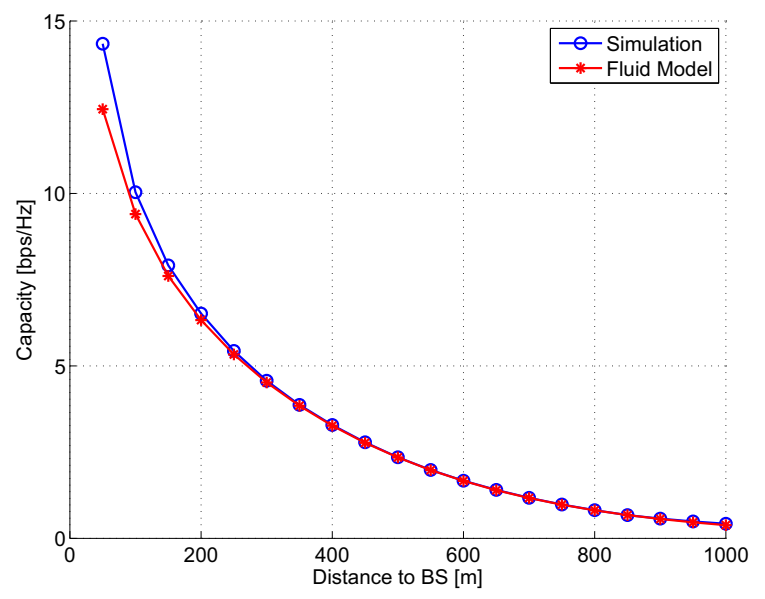

Figure 4: Capacity versus distance to BS for IFR with reuse 1 .

as:

$$
W=12 \int_{0}^{\pi / 6} \int_{0}^{R_{c} / \cos \theta} W_{u}(r) \rho_{u} r d r d \theta
$$

If $N_{u}$ is the number of users in a cell, user density is $\rho_{u}=$ $N_{u} /\left(2 \sqrt{3} R_{c}^{2}\right)$. Using Eq.6 and 4 , value of $\rho_{u}$ and variable transformation $r$ to $x$, user data rate is given as:

$$
D_{u}=\frac{\sqrt{3} W / 6}{N_{u} \int_{0}^{\pi / 6} \int_{0}^{1 / \cos \theta} \frac{x}{C_{I F R 1}(x)} d x d \theta},
$$

where $C_{I F R 1}(x)$ is given by Eq.3.

Since all users receive same data rate and there are $N_{u}$ users in the cell, total cell data rate is $D_{T, I F R 1}=N_{u} D_{u}$ and can be written using previous result as:

$$
D_{T, I F R 1}=\frac{\sqrt{3} W / 6}{\int_{0}^{\pi / 6} \int_{0}^{1 / \cos \theta} \frac{x}{C_{I F R 1}(x)} d x d \theta} .
$$

A worth noting observation regarding Eq.7 is that the total cell data rate neither depend upon the number of users in the cell nor upon the value of $R_{c}$.

The change of variables $\theta=z$ and $x=y / \cos z$, whose Jacobian is $\partial(\theta, x) / \partial(z, y)=1 / \cos z$, provides the equivalent equation:

$$
D_{T, I F R 1}=\frac{\sqrt{3} W / 6}{\int_{0}^{\pi / 6} \int_{0}^{1} \frac{y / \cos z}{\cos z C_{I F R 1}(y / \cos z)} d y d z} .
$$

To compare the above results with those of simulations, parameters of section 2 are used. One value of $\eta=3$ has been considered. Fig.4 shows the plot of capacity versus distance for both the fluid model (Eq.3) and simulation. The agreement between the two is apparent.

We now set the available network bandwidth to $W=$ $10 \mathrm{MHz}$ and the number of users per cell to $N_{u}=30$ in simulations. In Tab.1, total cell data rate $D_{T, I F R 1}$ with both the fluid model (Eq.7) and simulations, for various values of $\eta$, are given. The best agreement is for $\eta=2.7$, while the difference remains below $10 \%$ for $\eta$ between 2.6 and 3.2 . The user data rate $\left(D_{u}\right)$ can be easily obtained by dividing the total cell data rate (i.e., $D_{T, I F R 1}$ in this case) by number of users $\left(N_{u}\right)$ in the cell
Table 1: Total cell data rate $\left(D_{T, I F R 1}\right)$ versus $\eta$ (IFR reuse 1 , bandwidth $=10 \mathrm{MHz}$ )

\begin{tabular}{|l||c|c|c|}
\hline \multirow{2}{*}{$\eta$} & $D_{T, I F R 1}[\mathrm{Mbps}]$ & \multirow{2}{*}{ Difference } \\
\cline { 2 - 3 } & Fluid model & Simulation & \\
\hline \hline 2.5 & 6.62 & 7.43 & $10.9 \%$ \\
\hline 2.6 & 7.83 & 8.26 & $5.21 \%$ \\
\hline 2.7 & 9.01 & 9.08 & $0.77 \%$ \\
\hline 2.8 & 10.16 & 9.89 & $2.73 \%$ \\
\hline 3 & 12.4 & 11.6 & $6.9 \%$ \\
\hline 3.2 & 14.5 & 13.2 & $9.85 \%$ \\
\hline 3.3 & 15.5 & 13.9 & $11.51 \%$ \\
\hline
\end{tabular}

To avoid the complexity of calculating double integral, it is also possible to integrate $W_{u}(r)$ over a disk, whose area equals the hexagon area. Such a disk has a radius $R_{e}=$ $\sqrt{2 \sqrt{3} / \pi} R_{c}$. Using the above approach, total cell data rate can be approximated as:

$$
D_{T, I F R 1} \approx \frac{\sqrt{3} W / \pi}{\int_{0}^{a} \frac{x}{C(x)} d x} .
$$

For value of $\eta=3$ and $W=10 \mathrm{MHz}$, data rates obtained with Eq.7 and Eq. 8 are found to be $12.4 \mathrm{Mbps}$ and $12.6 \mathrm{Mbps}$ respectively with a difference of $1.6 \%$.

\subsection{IFR with Reuse $K$}

For IFR with reuse higher than one, analytical study is very similar to the previous one. The difference lies in the fact that only co-channel BS are considered in interference calculation and thus the half-distance between base stations and BS density have to be modified. As a consequence, previous analysis results are still valid provided that $R_{c}$ is replaced by $\sqrt{K} R_{c}$ and BS density is divided by $K$, i.e., $\rho_{B S}$ is replaced by $\rho_{B S} / K$. Hence using Eq.1 and this new half distance between BS, SINR is given as:

$$
\gamma(r)=\frac{r^{-\eta}(\eta-2)}{2 \pi \cdot \frac{\rho_{B S}}{K}\left(2 \sqrt{K} R_{c}-r\right)^{2-\eta}} .
$$

Using the same distance normalization as before (leading to the transformation of variable $r$ to $x$ ) and after few manipulations, SINR can be written as:

$$
\gamma_{I F R K}(x)=\frac{K \sqrt{3}}{\pi}(\eta-2)(2 \sqrt{K}-x)^{-2}(2 \sqrt{K} / x-1)^{\eta} .
$$

Hence capacity (in bps/Hz) for IFR reuse $K$ can be given as:

$$
C_{I F R K}(x)=\log _{2}\left[1+\gamma_{I F R K}(x)\right] .
$$

To validate above approach, reuse 3 is considered as an example. Plot of SINR and capacity versus distance for reuse 3 case, for both fluid model and simulation, are shown in Fig.5 and 6 respectively. As expected both SINR and capacity in $\mathrm{bps} / \mathrm{Hz}$ are higher than for reuse 1 . However, bandwidth per cell equals one third the network bandwidth. Again, both analysis and simulation provide similar results. The fluid model is thus accurate not only for reuse 1 networks but also for higher reuse factors provided the parameters are adjusted.

For total cell data rate, Eq.7 is still valid provided that $C_{I F R 1}(x)$ is replaced by $C_{I F R 3}$ (Eq.11) and cell bandwidth is divided by 3 . Values of total cell data rate for fluid model 


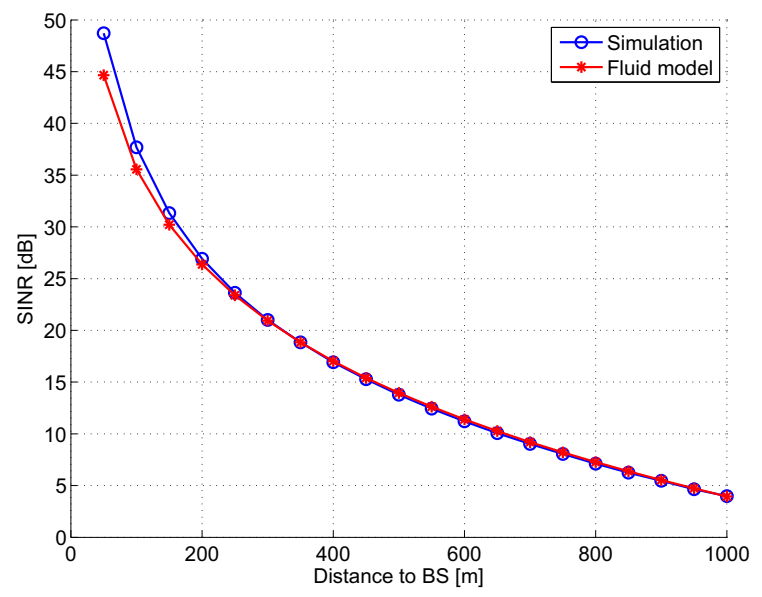

Figure 5: SINR versus distance to BS for IFR with reuse 3.

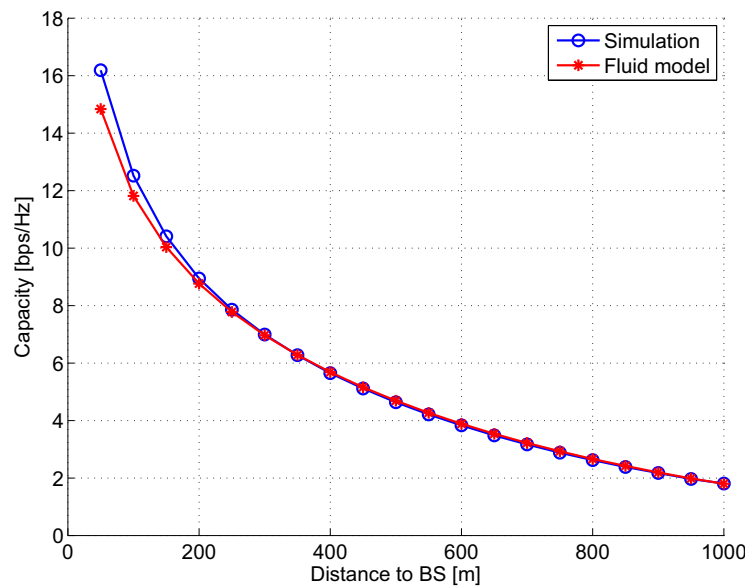

Figure 6: Capacity versus distance to BS for IFR with reuse 3 .

and simulations are shown in Tab.3.2. Again, the best agreement is obtained for $\eta=2.7$ and the difference remains below $10 \%$ for $\eta$ between 2.6 and 3.5.

\section{FRACTIONAL FREQUENCY REUSE (FFR)}

An example of FFR scenario is depicted in Fig.7. As can be seen in the figure, cell space is divided into two regions, inner and outer. Inner region is a circular disc with radius $R_{0}$ and rest of the hexagon space forms the outer region. Bandwidth is allocated to inner and outer in a way that former incorporates frequency reuse 1 while the latter applies frequency reuse 3. As can be seen in Fig.7, the network bandwidth $W$ is equal to $W_{0}+W_{1}+W_{2}+W_{3}$. It is also considered that $W_{1}=W_{2}=W_{3}$. In this section, we compute SINR and capacity as functions of the distance to the $\mathrm{BS}$, derive the total cell data rate assuming a scheduler fair in throughput, and we estimate the optimized inner region radius.
Table 2: Total cell data rate $\left(D_{T, I F R 3}\right)$ versus $\eta$ (IFR reuse $3, \mathbf{B W}=10 / 3 \mathrm{MHz}$ )

\begin{tabular}{|l||c|c|c|}
\hline \multicolumn{1}{|l||}{$\eta$} & \multicolumn{2}{|c|}{$D_{T, I F R 3}[\mathrm{Mbps}]$} & \multirow{2}{*}{ Difference } \\
\cline { 2 - 3 } & Fluid model & Simulation & \\
\hline \hline 2.5 & 6.4 & 7.2 & $11.11 \%$ \\
\hline 2.6 & 7.55 & 7.95 & $5.03 \%$ \\
\hline 2.7 & 8.66 & 8.73 & $0.8 \%$ \\
\hline 2.8 & 9.74 & 9.55 & $1.99 \%$ \\
\hline 3 & 11.8 & 11.2 & $5.36 \%$ \\
\hline 3.1 & 12.8 & 12.0 & $6.67 \%$ \\
\hline 3.2 & 13.8 & 12.8 & $7.81 \%$ \\
\hline 3.5 & 16.7 & 15.2 & $9.87 \%$ \\
\hline
\end{tabular}

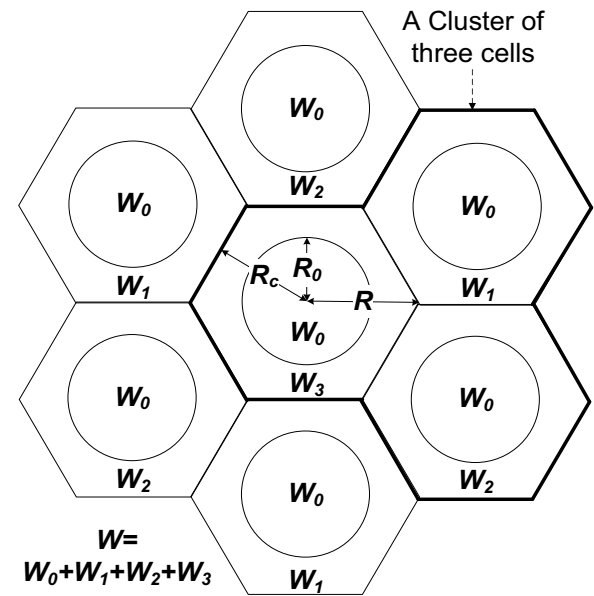

Figure 7: Fractional Frequency Reuse (FFR) Case. Bandwidth $W_{0}$ has been employed using frequency reuse 1 while $W_{1}, W_{2}$ and $W_{3}$ follow frequency reuse 3.

First, let us consider the inner circular region. Since for this region, frequency reuse is 1 , the expression of SINR is given by Eq.1 or equivalently by Eq.2. Once again, it is considered that users are uniformly distributed in the cell space with $\rho_{u}=N_{u} / 2 \sqrt{3} R_{c}^{2}$. Since it is a circular region, the elementary area $d s$ of Eq.5 can be simply taken as $2 \pi r d r$ and the bandwidth of inner region is given as:

$$
W_{0}=\int_{0}^{R_{0}} W_{u}(r) \rho_{u} 2 \pi r d r .
$$

After replacing $\rho_{u}$ by its value, transformation of variable $r$ to $x$ and using Eq.4, we get

$$
W_{0}=\frac{\pi}{\sqrt{3}} D_{u} N_{u} \int_{0}^{R_{0} / R_{c}} \frac{x}{C_{I F R 1}(x)} d x,
$$

where $C_{I F R 1}(x)$ is given by Eq.3. Let $I_{0}$ be the integral in the previous expression, so that $W_{0}=\pi / \sqrt{3} I_{0} D_{u} N_{u}$.

Let us now consider the outer area which applies reuse 3 . SINR for reuse 3 is given by Eq.9 or equivalently by Eq.10. In order to calculate the total bandwidth used in the outer region, double integral used in Eq.6 is applied with change of limits and replacing $W_{u}(r)$ by $W_{1, u}(r)$ we get:

$$
W_{1}=12 \int_{0}^{\pi / 6} \int_{R_{0}}^{R_{c} / \cos \theta} W_{1, u}(r) \rho_{u} r d r d \theta,
$$




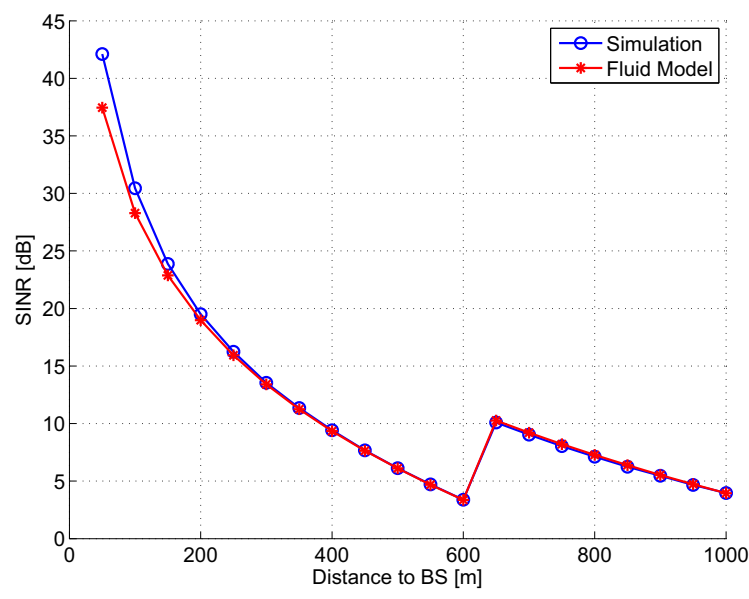

Figure 8: SINR versus distance to BS for FFR with $R_{0}=0.7 R_{c}$.

where $W_{1, u}(r)$, assuming fairness in throughput, is given as:

$$
W_{1, u}(r)=D_{u} C_{I F R 3}(r) .
$$

After replacing $\rho_{u}$ by its value, transformation of variable $r$ to $x$ and using Eq.13, we get

$$
W_{1}=\frac{6}{\sqrt{3}} D_{u} N_{u} \int_{0}^{\pi / 6} \int_{R_{0} / R_{c}}^{1 / \cos \theta} \frac{x}{C_{I F R 3}(x)} d x d \theta .
$$

Let $I_{1}$ be the double integral in the previous expression, so that $W_{1}=6 / \sqrt{3} I_{1} D_{u} N_{u}$.

Considering the fact that total network bandwidth is $W$ with $W=W_{0}+W_{1}+W_{2}+W_{3}$ and $W_{1}=W_{2}=W_{3}$ we can write:

$$
W=W_{0}+3 \times W_{1} .
$$

Finally using Eq.12, 14, 15 and keeping in view that $D_{T}=$ $D_{u} N_{u}$ we get expression of the total cell data rate $D_{T, F F R}$ for FFR case:

$$
D_{T, F F R}=\frac{\sqrt{3} W}{\pi I_{0}+18 I_{1}} .
$$

SINR and capacity versus distance with $R_{0}=0.7 R_{c}$ for fluid model and simulation are given in Fig. 8 and 9 respectively. The results follow the previous positive trend, i.e., fluid model and simulations are in conformity.

Total cell data rate calculation also shows that fluid model and simulation differ by $5.6 \%$ with values of $13.2 \mathrm{Mbps}$ and 12.5 Mbps respectively for $10 \mathrm{MHz}$ of network bandwidth. Fig.10 shows the total cell data rate as a function of the inner cell radius $R_{0}$. Both fluid model and simulations provide an optimum value of approximately $757 \mathrm{~m}$.

\section{TWO LEVEL POWER CONTROL (TLPC)}

In previous section, we discussed the concept of FFR in OFDMA and we have shown that SINR could be improved by using a reuse 3 pattern in cell outer regions. With FFR it is however not possible to use full network bandwidth in a cell, which reduces the overall cell capacity.

To overcome this drawback, it is possible to adopt a reuse 1 pattern while using a two level power control (TLCP)

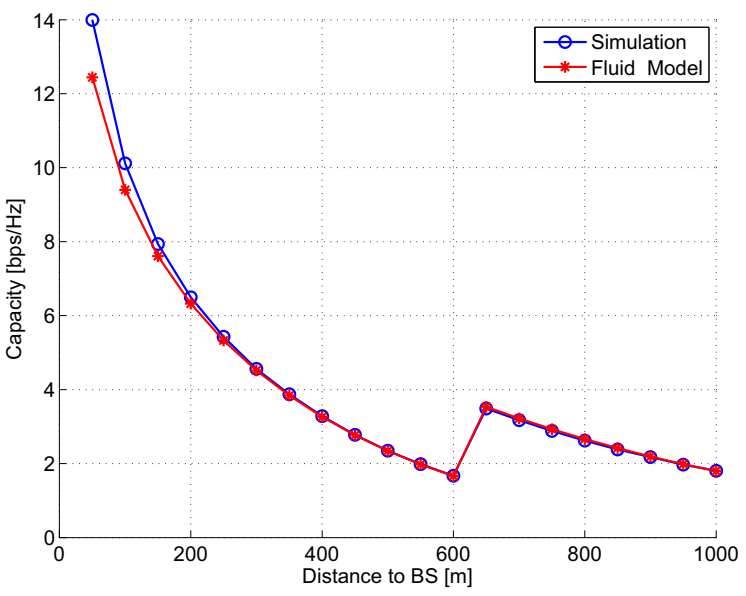

Figure 9: Capacity versus distance to BS for FFR with $R_{0}=0.7 R_{c}$.

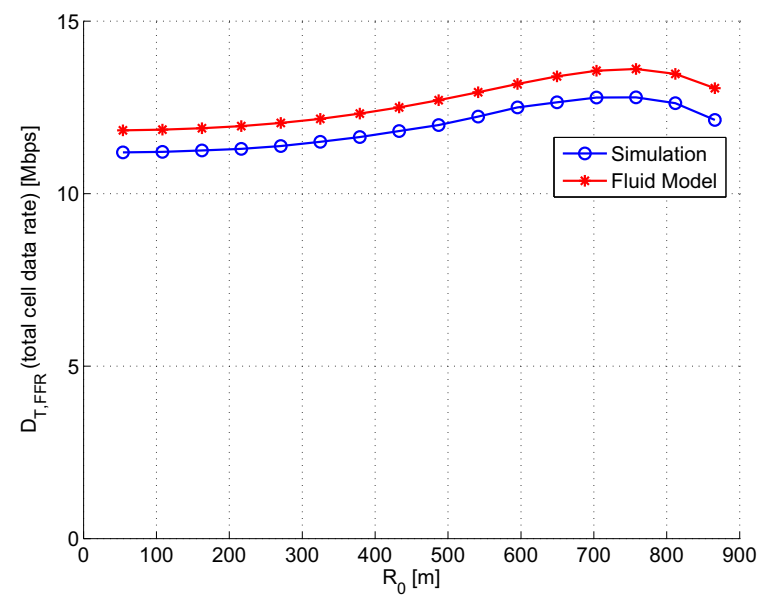

Figure 10: $D_{T, F F R}$ (total cell data rate) versus radius of inner region for FFR scheme. Maximum value occurs at $R_{0}=757 \mathrm{~m}$ approx.

mechanism to improve the radio quality in the outer region. The TLPC scheme is shown in Fig.11. Total bandwidth in a cell (equal to network bandwidth) is divided into three equal parts: two parts allocated to inner region and one to the outer region. The output power per subcarrier in the inner region is $P_{i}$ and that in the outer region is $P_{o}$. These two values of power are related as: $P_{o}=\delta P_{i}$, such that $\delta \geq 1$. The three spectrum parts $W_{1}, W_{2}$ and $W_{3}$ alternates from cell to cell in such a way that there is a pseudo-reuse 3 scheme between outer regions. Neighboring cells contribute in fact to interference in the outer region but with a reduced power $P_{i}$. The consequence is that the total network bandwidth is used in every cell but interference is expected to be reduced in outer regions.

Let us calculate SINR for inner and outer region of this two level power control network. For a user in the outer region (using e.g. $W_{3}$ in the center cell in Fig.11), we divide the interference into two categories. One is from the cells 


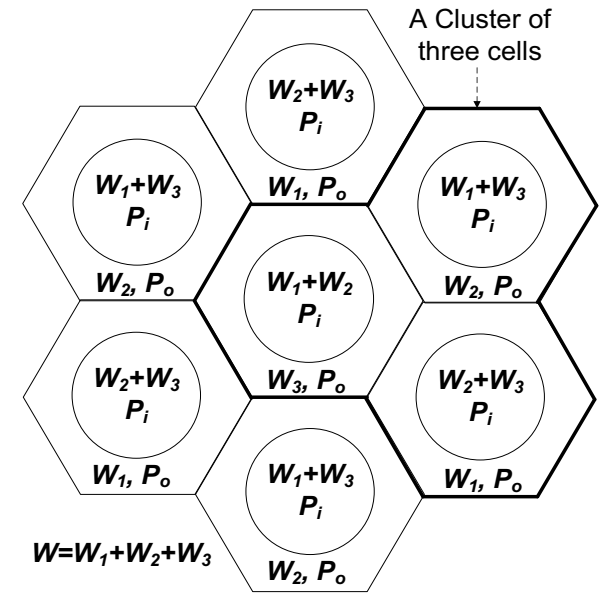

Figure 11: Two level power control case. Bandwidth $W$ is partitioned into three equal parts i.e., $W_{1}=$ $W_{2}=W_{3}$

using same sub-carriers in the outer region and we represent it by $I_{\text {outer }}$. Other is from cells using same sub-carriers in the inner region (neighboring cells) and is represented by $I_{\text {inner. }}$ Then, SINR for a subcarrier in the outer region can be written as:

$$
\gamma_{T L P C, \text { outer }}(r)=\frac{P_{o} A r^{-\eta}}{I_{\text {inner }}+I_{\text {outer }}} .
$$

With slight change of form, the above expression can be rewritten as:

$$
\frac{1}{\gamma_{T L P C, \text { outer }}}=\frac{I_{\text {inner }}}{P_{o} A r^{-\eta}}+\frac{I_{\text {outer }}}{P_{o} A r^{-\eta}} .
$$

In order to find the values of $I_{\text {inner }}$ and $I_{\text {outer }}$, consider that $\mathcal{B}_{\text {outer }}$ represents the set of BS causing $I_{\text {outer }}$. For a user $u$ in outer region of a cell $b, I_{\text {outer }}$ is given as:

$$
I_{\text {outer }}=P_{o} A \sum_{j=1, j \in \mathcal{B}_{\text {outer }}}^{N_{B S}} r_{j, u}^{-\eta} .
$$

As outer regions of $\mathrm{BS}$ in $\mathcal{B}_{\text {outer }}$ form together a reuse 3 scheme, the second term of right hand side of Eq.17 is simply $1 / \gamma_{I F R 3}$.

Adding up the interference from all network cells, $I_{\text {inner }}$ can be written as:

$$
I_{\text {inner }}=P_{i} A \sum_{j=1, j \neq b}^{N_{B S}} r_{j, u}^{-\eta}-P_{i} A \sum_{j=1, j \in \mathcal{B}_{\text {outer }}}^{N_{B S}} r_{j, u}^{-\eta} .
$$

Thus, considering $\delta=P_{o} / P_{i}$ :

$$
\frac{I_{\text {inner }}}{P_{o} A r^{-\eta}}=\frac{1}{\delta} \frac{1}{\gamma_{I F R 1}}-\frac{1}{\delta} \frac{1}{\gamma_{I F R 3}} .
$$

Combining previous results, we can rewrite Eq.17 as:

$$
\frac{1}{\gamma_{T L P C, \text { outer }}}=\frac{1}{\delta} \frac{1}{\gamma_{I F R 1}}+\left(1-\frac{1}{\delta}\right) \frac{1}{\gamma_{I F R 3}} .
$$

Now we find out SINR expression for inner region. Consider the central cell of Fig.11, in which $W_{1}$ and $W_{2}$ are allocated to center region and $W_{3}$ is used in the outer region. A user in inner region will be allocated a subcarrier

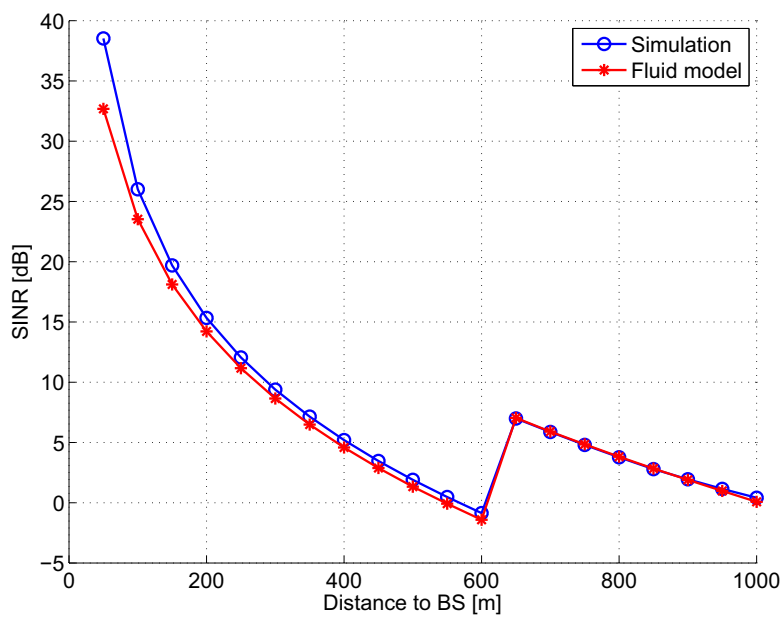

Figure 12: SINR versus distance to BS for TLPC scheme with $R_{0}=0.7 R_{c}$ and $\delta=5$.

that will either belong to $W_{1}$ or $W_{2}$. If we look at the bandwidth utilized in the six neighbouring cells of center cell, we notice that out of six, three are transmitting on the same subcarrier with power $P_{o}$, while other three with $P_{i}$. Hence, SINR for this inner region subcarrier can be approximated while considering that neighboring cells transmit with average power $\left(P_{i}+P_{o}\right) / 2$ and

$$
\gamma_{T L P C, \text { inner }}(r) \approx \frac{P_{i} A r_{b, u}^{-\eta}}{\frac{P_{o}+P_{i}}{2} A \sum_{j=1, j \neq b}^{N_{B S}} r_{j, u}^{-\eta}}=\frac{2}{1+\delta} \gamma_{I F R 1} .
$$

Using the values of SINR for outer and inner region, Shannon capacities per subcarrier for two regions are given in Eq.23 and 24 .

$$
\begin{aligned}
& C_{T L P C, \text { inner }}=\log _{2}\left(1+\gamma_{T L P C, \text { inner }}\right), \\
& C_{T L P C, \text { outer }}=\log _{2}\left(1+\gamma_{T L P C, \text { outer }}\right) .
\end{aligned}
$$

To verify the above results, SINR and capacity versus distance (with $R_{0}=0.7 R_{c}$ and $\delta=5$ ) are given in Fig.12 and 13 respectively. In order to calculate data rate per user for inner and outer region, we assume that in Fig.11 $W_{1}=W_{2}=W_{3}$. Using the similar approach of section 4 , we can write:

$$
\begin{aligned}
W_{1}+W_{2}= & \frac{2 W}{3}=\frac{\pi}{\sqrt{3}} D_{u, \text { inner }} N_{u} \times \\
& \int_{0}^{R_{0} / R_{c}} \frac{x d x}{C_{T L P C, \text { inner }}(x)},
\end{aligned}
$$

and

$$
\begin{aligned}
W_{3}= & \frac{W}{3}=\frac{6}{\sqrt{3}} D_{u, \text { outer }} N_{u} \times \\
& \int_{0}^{\pi / 6} \int_{R_{0} / R_{c}}^{1 / \cos \theta} \frac{x}{C_{T L P C, \text { outer }}(x)} d x d \theta .
\end{aligned}
$$

Using above two equations, we can write the ratio between data per user for inner and outer region as:

$$
\frac{D_{u, \text { inner }}}{D_{u, \text { outer }}}=\frac{12}{\pi} \frac{\int_{0}^{\pi / 6} \int_{R_{0} / R_{c}}^{1 / \cos \theta} \frac{x}{C_{\text {TLPC, outer }}(x)} d x d \theta}{\int_{0}^{R_{0} / R_{c}} \frac{x}{C_{T L P C, \text { inner }}(x)} d x} .
$$




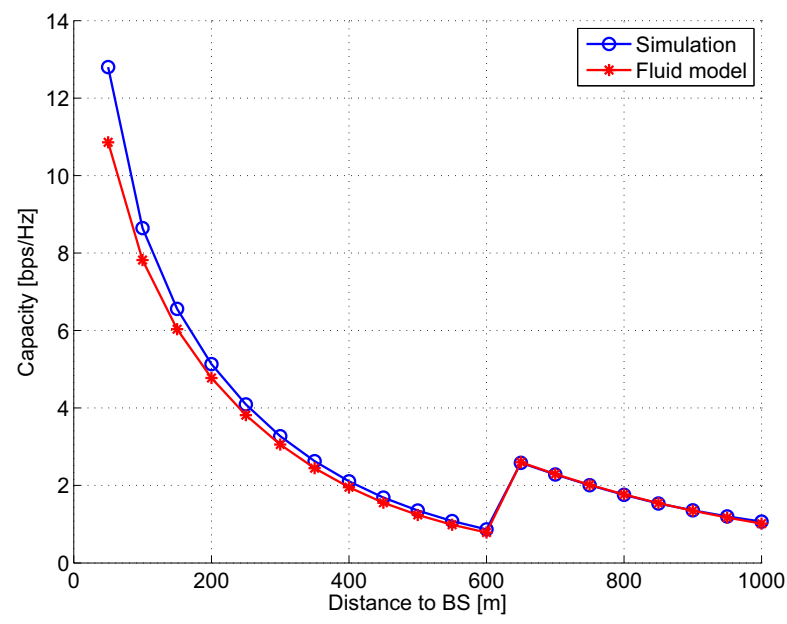

Figure 13: Capacity versus distance to BS for TLPC scheme with $R_{0}=0.7 R_{c}$ and $\delta=5$.

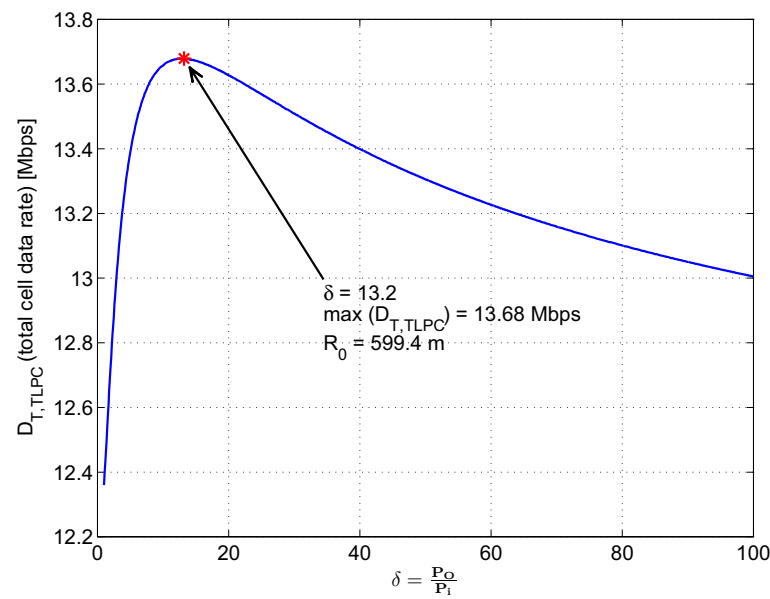

Figure 14: Total cell data rate versus different values of $\delta$ for TLPC scheme.

Now, if we assume a scheduler fair in throughput, $\frac{D_{u, \text { inner }}}{D_{u, \text { outer }}}$ should be equal to one. For a given value of $\delta$ there exists a unique value of $R_{0}$ for which above condition is satisfied. In Fig.14, total cell data rate satisfying fairness in throughput has been plotted for various values of $\delta$. It is clear that in the beginning, total cell data rate increases with increasing values of $\delta$. The total cell data rate attains its maximum value of $13.68 \mathrm{Mbps}$ for $\delta=13.2$. Beyond $\delta=13.2$ (or $11.2 \mathrm{~dB}$ ), the total cell data rate starts decreasing. The corresponding values of $R_{0}$ for these total cell data rates are given in Fig.15. The figure shows a decrease in the values of $R_{0}$ for increasing values of $\delta$. The value of $R_{0}$ corresponding to maximum value of cell data rate is $599.4 \mathrm{~m}$.

\section{COMPARISON OF IFR, FFR AND TLPC}

In previous sections, we have established through validation that analytical approach based on the fluid model can

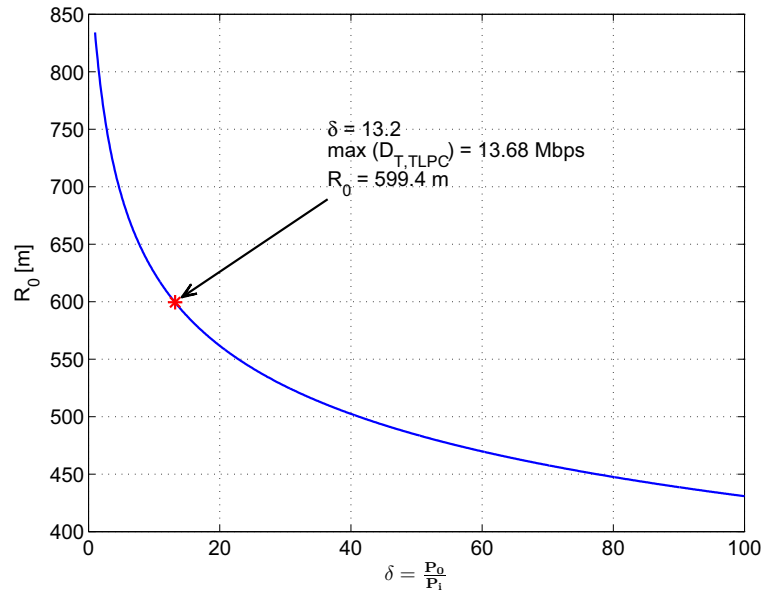

Figure 15: $R_{0}$ (radius of inner region that guarantees fairness in throughput among users) versus different values of $\delta$ for TLPC scheme.

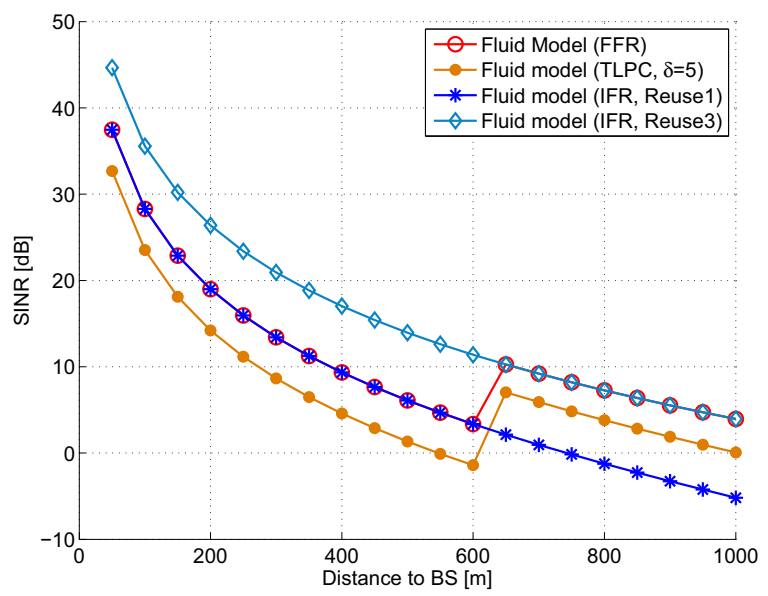

Figure 16: SINR versus distance to BS for three reuse schemes.

be used for IFR, FFR and TLPC schemes. In this section, we present a comparison between these three schemes.

If we look at Fig.16 and 17, it can be deduced that IFR with reuse 3 shows the best performance in terms of SINR values. IFR reuse 1 is much lower than IFR reuse 3 in terms of radio quality. FFR exactly follows IFR reuse 1 curve until $R_{0}$ and IFR reuse 3 beyond. Compared to IFR reuse 1, TLPC improves SINR in outer region at the expense of a degraded radio quality in inner region.

We now compare total cell data rates under the assumption of a scheduler fair in throughput. Network bandwidth is $10 \mathrm{MHz}$. Value of $R_{0}$ for FFR is $757 \mathrm{~m}$ which was found to be optimal (cf. section 4). In case of TLPC, values of $R_{0}$ is the one that satisfies fairness in data rate condition for a given value of $\delta$. Three different values of $\delta$ have been considered. Results of cell data rate are listed in Tab.3. These results show that IFR reuse 3 touches the lowest performance although SINR values are greater. This is due to the fact that 


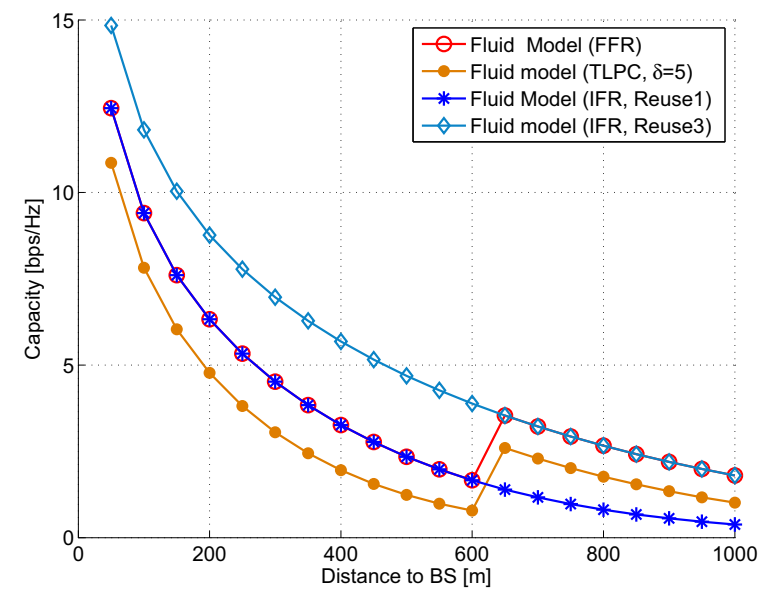

Figure 17: Capacity versus distance to BS for three reuse schemes.

Table 3: Total cell data rate $\left(D_{T}\right)$ comparison of three schemes.

\begin{tabular}{|l||c|}
\hline & $\mathbf{D}_{\mathbf{T}}[\mathrm{Mbps}]$ \\
\hline \hline FFR, $\mathbf{R}_{\mathbf{0}}=\mathbf{7 5 7} \mathbf{~} \mathbf{~}$ & 13.61 \\
\hline TLPC, $\delta=\mathbf{1}, \mathbf{R}_{\mathbf{0}}=\mathbf{8 3 4 . 2} \mathbf{~ m}$ & 12.36 \\
\hline TLPC, $\delta=\mathbf{5}, \mathbf{R}_{\mathbf{0}}=\mathbf{8 1 0} \mathbf{~ m}$ & 12.50 \\
\hline TLPC, $\delta=\mathbf{1 3 . 2}, \mathbf{R}_{\mathbf{0}}=\mathbf{5 9 9 . 4} \mathbf{~ m}$ & 13.68 \\
\hline IFR, Reuse $\mathbf{1}$ & 12.3 \\
\hline IFR, Reuse $\mathbf{3}$ & 11.8 \\
\hline
\end{tabular}

cell utilization of network bandwidth is lower compared to other schemes. Numerical results clearly indicate that FFR and TLPC with $\delta=13.2$ have the best performance with total cell data rate of latter just ahead of the former.

\section{CONCLUSIONS}

In this paper, we have presented an analytical approach, based on the fluid model, for analyzing OFDMA based networks. We have shown that our proposed technique is very flexible and can be used in different frequency reuse scenarios. We have introduced expressions of SINR and cell data rate for IFR, FFR and TLPC schemes. We have also validated our technique by comparing its results with those obtained from Monte Carlo simulations. Time required to obtain results with our analytical technique is however much shorter. We have shown that our proposed technique gives a fairly good performance for $\eta$ values between 2.6 and 3.5 which is a range found in most of practical scenarios. A brief comparison of above three schemes is also provided. It has been shown that FFR and TLPC schemes with appropriate settings of inner region radius and power ratio provide the best performance when a scheduler fair in throughput is assumed. As further work, we intend to take into account the effect of subcarrier diversity, shadowing and other scheduling schemes (e.g., opportunist, proportional fair).

\section{ACKNOWLEDGMENTS}

This work was jointly carried out by TELECOM ParisTech and France Telecom R\&D within the framework of project SYSTEMATIC/URC administrated by Île-de-France (Paris Region).

Authors would also like to thank Trung Dung Nguyen, student at ENST, whose project [11] under the supervision of Philippe Godlewski formed the first step in carrying out this work.

\section{REFERENCES}

[1] Mobile WiMAX-Part II: A Comparative Analysis. Technical report, WiMAX Forum, May 2006.

[2] C. He, F. Liu, H. Yang, C. Chen, H. Sun, W. May, and J. Zhang. Co-channel Interference Mitigation in MIMO-OFDM System. In Proc. of IEEE WiCom, Sept. 2007.

[3] H. Jia, Z. Zhang, G. Yu, P. Cheng, and S. Li. On the Performance of IEEE 802.16 OFDMA System under Different Frequency Reuse and Subcarrier Permutation Patterns. In Proc. of IEEE ICC, June 2007.

[4] J.-M. Kelif and E. Altman. Downlink Fluid Model of CDMA Networks. In Proc. of IEEE VTC Spring, May 2005.

[5] J.-M. Kelif, M. Coupechoux, and P. Godlewski. Spatial Outage Probability for Cellular Networks. In Proc. of IEEE GLOBECOM, Nov. 2007.

[6] J.-M. Kelif, M. Coupechoux, and P. Godlewski. Effect of Shadowing on Outage Probability in Fluid Cellular Networks. In Proc. of WiOpt, Apr. 2008.

[7] G. Kulkarni, S. Adlakha, and M. Srivastava. Subcarrier Allocation and Bit Loading Algorithms for OFDMA-Based Wireless Networks. IEEE Trans. on Mobile Computing, 4(6), Dec. 2005.

[8] G. Liu, J. Zhu, F. Jiang, B. Zhou, Y. Wang, and P. Zhang. Initial Performance Evaluation on TD-SCDMA Long Term Evolution System. In Proc. of IEEE VTC Spring, May 2006.

[9] M. C. Necker. Coordinated Fractional Frequency Reuse. In Proc. of ACM MSWiM, Oct. 2007.

[10] M. C. Necker. Local Interference Coordination in Cellular 802.16e Networks. In Proc. of IEEE VTC Fall, Oct. 2007.

[11] T. D. Nguyen and P. Godlewski. Capacité OFDMA. Technical report, ENST (Télécom ParisTech), 2006.

[12] K. Ramadas and R. Jain. WiMAX System Evaluation Methodology. Technical report, WiMAX Forum, Jan. 2007.

[13] C. Tarhini and T. Chahed. On Capacity of OFDMA-based IEEE802.16 WiMAX Including Adaptive Modulation and Coding (AMC) and Inter-cell Interference. In Proc. of IEEE Workshop on LANMAN, Jun. 2007.

[14] V. Erceg, L.J.Greenstein, et.al. An Empirically Based Path Loss Model for Wireless Channels in Suburban Environments. In IEEE Journal on Selected Areas in Communications, July 1999.

\section{APPENDIX \\ Fluid Model in OFDMA Networks}

In this section, we recall the main results of the fluid model and derive the closed-form formula for $S I R$ per sub-carrier. 


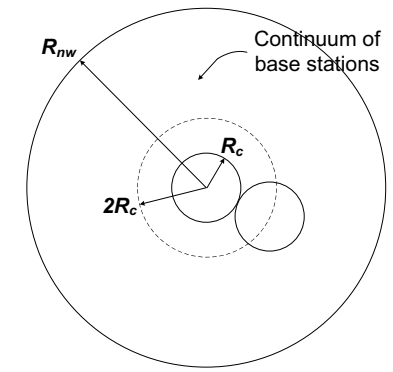

Figure 18: Network and cell of interest in the fluid model; the distance between two $\mathrm{BS}$ is $2 R_{c}$ and the network is made of a continuum of base stations.

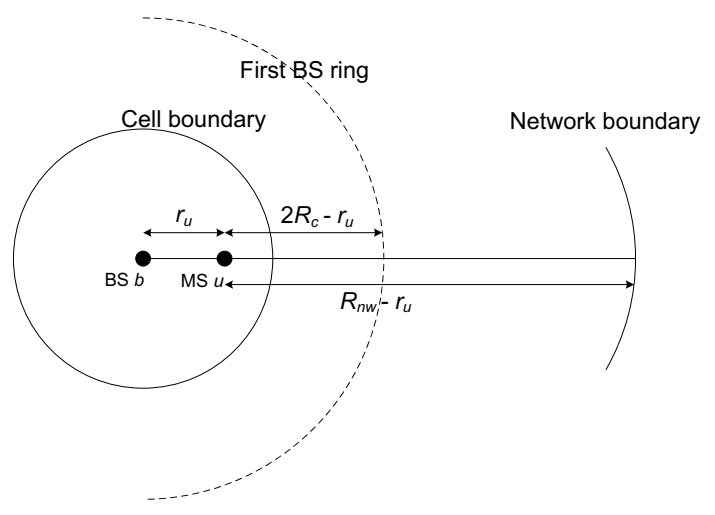

Figure 19: Integration limits for interference computation.

The key modeling step of the fluid model is replacing a given fixed finite number of BS by an equivalent continuum of transmitters which are spatially distributed in the network. This means that the transmitting power is now considered as a continuum field all over the network. In this context, the network is characterized by a user density $\rho_{u}$ and a base station density $\rho_{B S}[4]$. We assume that users and BS are uniformly distributed in the network, so that $\rho_{u}$ and $\rho_{B S}$ are constant. We assume also that all base stations have the same output power per sub-carrier $P_{T x}$.

We focus on a given cell, on a generic sub-carrier and consider a round shaped network around this central cell with radius $R_{n w}$. The half distance between two base stations is $R_{c}$ (see Fig.18 in case of reuse 1 ).

Let's consider a user $u$ at a distance $r_{u}$ from its serving base station $b$. Each elementary surface $z d z d \theta$ at a distance $z$ from $u$ contains $\rho_{B S} z d z d \theta$ base stations which contribute to $I_{e x t, u}$. Their contribution to the external interference is thus $\rho_{B S} z d z d \theta P_{T x} A z^{-\eta}$. We approximate the integration surface by a ring with center $u$, inner radius $2 R_{c}-r_{u}$, and outer radius $R_{n w}-r_{u}$ (see Fig.19).

$$
\begin{gathered}
I_{e x t, u}=\int_{0}^{2 \pi} \int_{2 R_{c}-r_{u}}^{R_{n w}-r_{u}} \rho_{B S} P_{T x} A z^{-\eta} z d z d \theta \\
=\frac{2 \pi \rho_{B S} P_{T x} A}{\eta-2}\left[\left(2 R_{c}-r_{u}\right)^{2-\eta}-\left(R_{n w}-r_{u}\right)^{2-\eta}\right] .
\end{gathered}
$$

So, the SINR $\gamma_{u} \approx S_{b, u} / I_{e x t, u}=P_{T x} A r_{u}^{-\eta} / I_{e x t, u}$ can be expressed by:

$$
\gamma_{u}=\frac{r_{u}^{-\eta}(\eta-2)}{2 \pi \rho_{B S}\left[\left(2 R_{c}-r_{u}\right)^{2-\eta}-\left(R_{n w}-r_{u}\right)^{2-\eta}\right]} .
$$

Note that $\gamma_{u}$ does not depend on the BS output power. This is due to the fact that we assumed an homogeneous network and so all base stations emit the same power on a given sub-carrier. In this model, $\gamma$ only depends on the distance $r$ from the BS and can be defined in each location, so that we can write $\gamma$ as a function of $r, \gamma(r)$. If the network is large, i.e., $R_{n w}$ is big in front of $R_{c}, \gamma_{u}$ can be further approximated by:

$$
\gamma_{u}=\frac{r_{u}^{-\eta}(\eta-2)}{2 \pi \rho_{B S}\left(2 R_{c}-r_{u}\right)^{2-\eta}} .
$$

The fluid model and the traditional hexagonal model are two simplifications of the reality. None is a priori better than the other but the latter is widely used, especially for dimensioning purposes. That is the reason why comparisons are performed throughout the paper.

Reference [5] has shown that the considered network size can be finite and can be chosen to characterize each specific local network's environment. This model thus allows us to do the analysis adapted to each zone while taking into account considered zone's specific parameters. Moreover, it can be noticed that the fluid model can be used even for great distances between the base stations. 